\title{
Mathematical model approach to understand the ecological effect under chronic irradiation
}

\author{
I. Kawaguchi, M. Doi and S. Fuma \\ Research Center for Radiation Protection, National Institute of Radiological Sciences \\ 4-9-1 Anagawa, Inage-ku, Chiba 263-8555, Japan
}

\begin{abstract}
Although aim of the environmental protection is conservation of ecosystem, there are only a few studies focusing on the effect of radiation on ecosystem. To understand the ecological effect of irradiation, microbial ecosystem, "microcosm", which contains minimum components of ecosystem such as producer, consumer and decomposer, is useful because the natural ecosystem is too complex. The microcosm consists of three species, i.e. Euglena (producer), Tetrahymena (consumer), and E. coli (decomposer). The mathematical model and computer simulation model were also developed to understand the mechanism of ecological interaction using the results of acute exposure experiments of the microcosm, and we predicted Tetrahymena, which is the most radio-resistant among the constituent species, would be most sensitive in the chronically irradiated microcosm as a result of an indirect effect due to population decrease in E. coli. Recently we started chronic exposure experiments. The microcosms were irradiated with $\gamma$-rays at dose rate of $1.2 \mathrm{~Gy} /$ day, $5 \mathrm{~Gy} / \mathrm{day}, 10 \mathrm{~Gy} / \mathrm{day}$ and $23 \mathrm{~Gy} /$ day. From preliminary results, we found that the prediction from the models was different from the experimental results. Therefore, in this study, we improved our mathematical model and discuss the difference between the model and experiments.
\end{abstract}

\section{INTRODUCTION}

Almost all of researches which are studied about the impact of radiation to the environment or ecosystem are focused on the individual level and are not considered interaction of inter-species or intra-species. However, there are underlying large difficulty in studying the natural ecosystem directly, because the real ecosystem consists of huge species and construct complex interaction network between the species. Microcosm is the one of the most useful tool to study ecosystem, which consists of minimum component of natural ecosystem and can observe and simulate interaction among the species in laboratory level. We have been studying the effect of the irradiation to ecosystem using the microcosm which developed by Kawabata et al. [1]. The microcosm consists of three species, Flagellate algae Euglena as a producer, ciliate protozoa Tetrahymena as a consumer and bacteria Escherichia Coli as a decomposer. The interaction between the species in the microcosm is summarized in Figure 1. The microcosm is maintained by photoenergy which Euglena fixed by photosynthesis, E. coli consumes the products which released from Euglena, and Tetrahymena grazes E. coli directly [1-6].

The $50 \%$ lethal doses $\left(\mathrm{LD}_{50}\right)$ of acute exposure of single species have been reported in previous study [2], E. coli is the most sensitive species and Tetrahymena is the most resistant species to $\gamma$-irradiation. However, in the three species co-cultured microcosm, the extinction order is E. coli (the most sensitive species), Tetrahymena, and Euglena (the most resistant species). This result did not due to change the radiation sensitivity of Tetrahymena but caused indirectly by extinction of E.coli.

From the experimental results of acute irradiation, we developed the mathematical models and analyzed about the situation of chronic exposure [7, 8]. From the analysis of the models, we found that Tetrahymena who is the most radio-resistant species in single culture is the most radiation sensitive species and $E$. coli is the resistant to radiation rather than Tetrahymena. This result is robust when the interaction of the species is simple prey-predator type. 


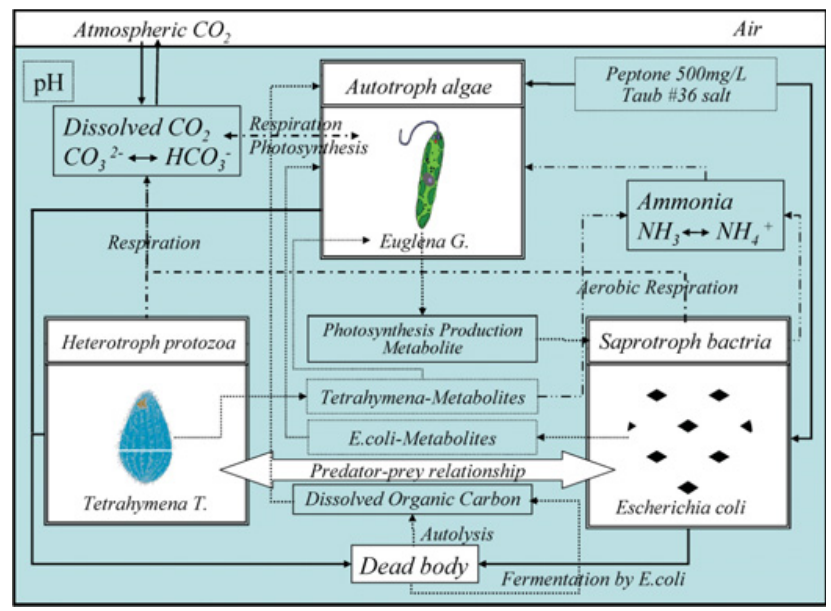

Figure 1. Schemes of interrelationships among microorganisms and constituent elements in Kawabata-microcosm taken from [1-5].

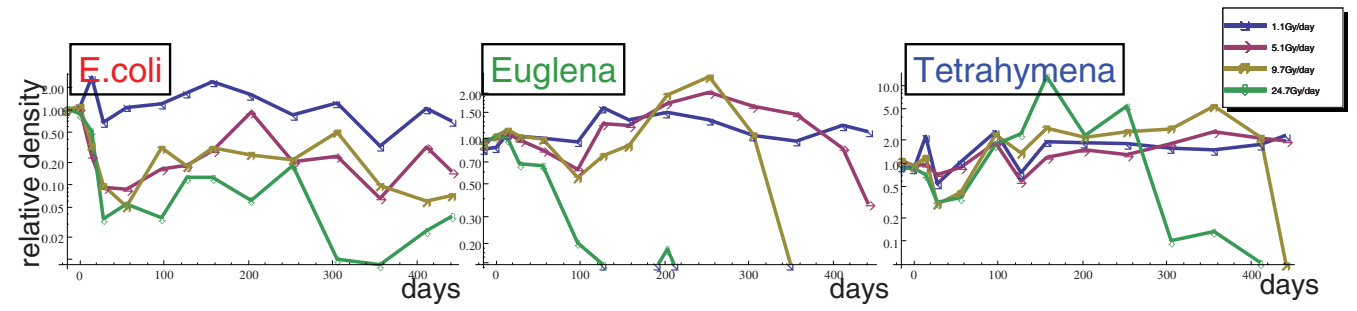

Figure 2. Summary of the experimental results of the chronically irradiated microcosm. The horizontal axis is time since onset of exposure, and vertical axis is the relative population density to the control.

Figure 2 showed the summary of the results of chronic exposure to the microcosm [8]. The microcosms were irradiated with $\gamma$-rays at dose rate of $1.2 \mathrm{~Gy} / \mathrm{day}, 5 \mathrm{~Gy} / \mathrm{day}, 10 \mathrm{~Gy} / \mathrm{day}$ and $23 \mathrm{~Gy} / \mathrm{day}$. From the experimental results of chronic exposure, population density of the E.coli decreases with dose rate, and Tetrahymena seems almost constant or slightly increased with dose rate increasing. These results are different from the model prediction, and imply that the interaction between Tetrahymena and E.coli is not simple prey-predator relationship. One explanation for this difference is that predation resistant phenotype existed in E. coli population. Nakajima and Kurihara [9] reported that the E. coli which has very long body length observed when $E$. coli is exposed to predation pressure. Therefore, in this study, we improved our model to include the predation resistant phenotype in E.coli population.

\section{THE MODEL}

We consider the E. coli have two phenotypes, normal phenotype and predation resistant phenotype, and we assumed those phenotypes were genetically distant. We assumed the resistant phenotype of E. coli escape from the predation, however, the growth rate of the resistant phenotype is lower than that of normal phenotype. We consider the population density of the normal phenotype, $N$, is increased by consuming the photosynthesis products from Euglena $(P)$ and decreased by predation. On the other hand, population density of resistant phenotype, $L$, also increases with consuming the products, however, decrement term by predation is omitted because of its resistant body form. The dynamics of 

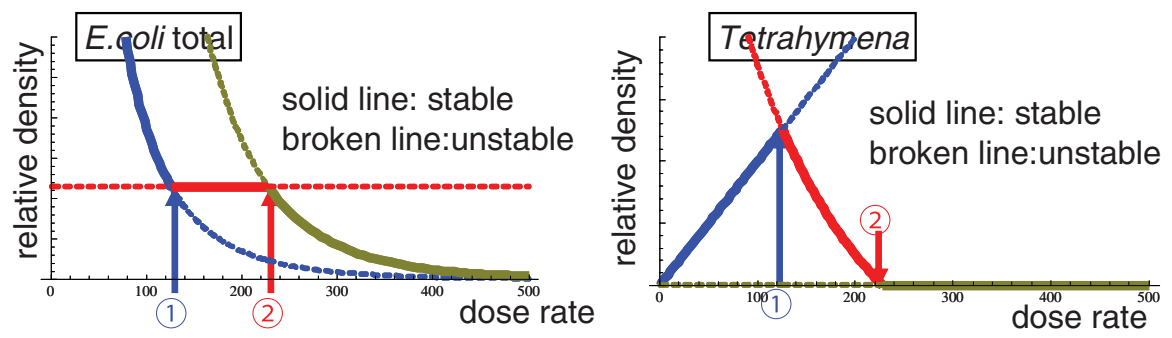

Figure 3. Summary of the analytical results of the model.

the system described as follows:

$$
\begin{aligned}
& \dot{E}=r_{E} E(1-E / K)-\alpha_{E} E \\
& \dot{P}=s E-a(N+L) P \\
& \dot{N}=r_{N} a N P-b N T-\alpha_{N} N \\
& \dot{L}=r_{L} a L P-\alpha_{L} L \\
& \dot{T}=r_{T} b N T-\alpha_{T} T
\end{aligned}
$$

where $E, N, L$ and $T$ denote population density of Eulgena, normal phenotype of E. coli, predationresistant phenotype E. coli and Tetrahymena, respectively. $r_{x}$ is the intrinsic growth rate of species $x$ ( $x=E, N, L$ or $T$ ), $K$ is the carrying capacity of Euglena, $s$ is the release rate of photosynthesis products from Euglena, $a$ is the predation rate of E. coli, $b$ is the predation rate of Tetrahymena, and $\alpha_{x}$ is the additional mortality of species $x$ due to the irradiation. According to the $\mathrm{LD}_{50}$ value of single species, we assumed the radiation sensitivity of E. coli is the highest in the microcosm and the lowest is Tetrahymena.

\section{RESULTS AND DISCUSSION}

From the analysis of equation (1), we found four equilibria in the system except a trivial equilibrium which is all species extinguished. We analyzed local stability of the equilibria and found that two or more stable equilibria could not coexist. We also found that the equilibrium which is coexisting of Euglena and resistant phenotype of E. coli was always unstable since we assumed that the growth rate of resistant phenotype is lower than the normal phenotype. Typical results of the model were summarized in Figure 3. The total population density of two phenotype in E.coli decreases with increasing the dose rate until threshold 1 which predation resistant $E$. coli goes extinct at. In this dose rate region, population density of Tetrahymena increases, because the predation resistant $E$. coli decreases with increasing dose rate, so that the normal phenotype obtains more products from the Euglena and increases the population density. Increment of the density of the normal $E$. coli results the increment of the density of predator species, Tetrahymena, and apparent population density of normal phenotype is balancing the increment due to gain of the products from Euglena and decrement by increasing predation pressure.

When the dose rate is larger than the threshold 1 and lower than the threshold 2, population density of $E$. coli stays constant, whereas Tetrahymena density monotonically decreases and equals to 0 at threshold 2. In this region, the relationship between E.coli and Tetrahymena is again simple preypredator model, therefore, the interpretation of the result is the same as previous our model.

From the results of the present model, in spite of the simplification of the model structure, the model qualitatively explained the experimental result. Therefore, emergence of the predation resistant phenotype in E. coli would have important role when radiation exposed chronically to microcosm. 
However, interaction among the species is still complex even if the microcosm consists of only three species. To understand the real dynamics of the system, more realistic modeling approach such as individual based model could show other cutting edges.

\section{References}

[1] Kawabata, Z., Matsui, K., Okazaki, K., Nasu, M., Nakano, N., Sugai, T. (1995), J. Protozool. Research, 5, 23-26.

[2] Fuma, S., Takeda, H., Miyamoto, K., Yanagisawa, K., Inoue, Y., Sato, N., Hirano, M., Kawabata, Z. (1998), Effects of $\gamma$-rays on the populations of the steady-state ecological microcosm, Int.J.Radiation biology, 74, 145-150.

[3] Fuma, S., Takeda, H., Miyamoto, K., Yanagisawa, K., Inoue, Y., Ishii, N., Sugai, K., Ishii, C., Kawabata, Z. (2001), Ecological evaluation of gadolinium toxicity compared with other heavy metals using an aquatic microcosm. Bulletin. Environmental Cont. and Toxicol., 66, 231-238.

[4] Fuma, S., Ishii, N., Takeda, H., Miyamoto, K., Yanagisawa, K., Ichimasa, Y., Saito, M., Kawabata, Z., Polikarpov, G.G. (2003), Ecological effects of various toxic agents on the aquatic microcosm in comparison with acute ionizing radiation. J. Environmental Radioactivity, 67, 1-14.

[5] Shikano, S., Kawabata, Z. (2000), Effect at the ecosystem level of elevated atmospheric $\mathrm{CO}_{2}$ in an aquatic microcosm. Hydrobiologia, 436, 209-216.

[6] Doi, M., and Kawaguchi, I. (2007), Ecological impacts of umbrella effects of radiation on the individual members. J. Environmental Radioactivity, 96, 32-38.

[7] Doi, M., Kawaguchi, I., Tanaka, N., Fuma, S., Ishii, N., Miyamoto, K., Takeda, H., Kawabata, Z. (2005), Model ecosystem approach to estimate community level effects of radiation. Radioprotection, Suppl. 1, 40, S913-S919.

[8] Fuma, S., Kawaguchi, I., Kubota, Y., Yoshida, S. Kawabata Z., and Polikarpov, G.G. Effects of chronic $\gamma$-irradiation on the aquatic microbial microcosm, submitted.

[9] Shikano S., Luckinbill L.S., and Kurihara Y. (1990) Changes of traits in a bacterial population associated with protozal predation. Micro. Ecol. 20, 75-84.

[10] Nakajima, T. and Kurihara, Y., (1994) Evolutionary changes of ecological traits of bacterial populations through predator-mediated competition., Oikos, 71, 24-34. 